\title{
Picos de concentração de poluição atmosférica na Região da Grande Vitória, ES, Brasil: uma aplicação da regressão logística
}

\author{
Atmospheric pollution concentration peaks in the Região da \\ Grande Vitória, ES, Brazil: an application of logistic regression
}

\author{
Wanderson de Paula Pinto ${ }^{1}$ \\ Valdério Anselmo Reisen ${ }^{2}$ \\ Edson Zambon Monte ${ }^{3}$
}

\begin{abstract}
Resumo
Este trabalho objetivou avaliar os impactos das variáveis meteorológicas temperatura, umidade relativa, velocidade do vento e precipitação na probabilidade de ocorrência de picos/episódios de concentração de poluentes na Região da Grande Vitória (RGV), Espírito Santo, Brasil, por meio do modelo Logit, para o período de 01/01/2012 a 31/12/2014. Os dados deste estudo foram do tipo séries temporais relativos às concentrações de $\mathrm{PTS}, \mathrm{MP}_{10}, \mathrm{SO}_{2}, \mathrm{CO}, \mathrm{NO}_{2}, \mathrm{O}_{3}$ e às variáveis meteorológicas (velocidade do vento, umidade relativa, precipitação pluvial e temperatura) obtidas junto ao Instituto Estadual de Meio Ambiente e Recursos Hídricos (IEMA). Conforme esperado, os resultados mostraram que os índices de concentrações na RGV estão associados às mudanças das variáveis meteorológicas. Entre as variáveis meteorológicas avaliadas, a velocidade do vento e a precipitação foram mais significativas na redução da probabilidade de ocorrência de classificação do ar como "não boa". O modelo Logit mostrou ser uma ferramenta estatística satisfatória para avaliar a qualidade do ar "não boa" da região de estudo.
\end{abstract}

Palavras-chave: Poluição atmosférica; modelo Logit; variáveis meteorológicas.

\begin{abstract}
This study aimed to evaluate the impacts of meteorological variables temperature, relative humidity, wind speed and precipitation in the probability of occurrence of pollutants concentration peaks/episodes in the Região da Grande Vitória (RGV), Espírito Santo, Brazil, using the Logit model, for period from 2012/01/01 to 2014/12/31. It were adopted time series of the pollutants PTS, $\mathrm{PM}_{10}, \mathrm{SO}_{2}, \mathrm{CO}, \mathrm{NO}_{2}, \mathrm{O}_{3}$ and meteorological variables (wind speed, relative humidity, rainfall and temperature)
\end{abstract}

\footnotetext{
1 Doutorando em Engenharia Ambiental pela Universidade Federal do Espírito Santo - UFES, Vitória, ES, Brasil. wandersondpp@gmail.com

2 Professor do Departamento de Economia e do Programa de Pós-Graduação em Economia e membro do Grupo de Pesquisa em Econometria da UFES, Vitória, ES, Brasil. edsonzambon@yahoo.com.br

3 Programa de Pós-Graduação em Engenharia Ambiental e Programa de Pós-Graduação em Economia, Departamento de Estatística, Universidade Federal do Espírito Santo, Vitória, ES, Brasil. valderioanselmoreisen@gmail.com

Artigo recebido em: 01/05/2017. Aceito para publicação em: 30/10/2018.
} 
obtained from the State Institute of Environment and Resources Water (IEMA). As expected, the results showed that the contents of the RGV concentrations are associated with changes of the meteorological variables. The results showed that the precipitation and the wind speed contributed significantly to the reduction of the probability of air quality "not good". The Logit model proved to be a satisfactory statistical tool for evaluating the air quality "not good" in the study region.

Keywords: Atmospheric pollution; Logit model; meteorological variables.

\section{Introdução}

A preocupação com os efeitos da poluição do ar veio com o crescimento industrial iniciado no período da Revolução Industrial que teve início na Inglaterra, em meados do século XVIII, devido à alguns episódios de alta concentração de poluentes. Episódios esses que causaram aumento do número de mortes em algumas cidades da Europa e dos Estados Unidos.

O primeiro episódio ocorreu em 1930, no vale do Meuse, na porção Belga, que provocou a morte de 60 pessoas. Anos mais tarde, em 1948, um episódio semelhante ocorreu durante os últimos cinco dias do mês de outubro, na cidade de Donora, Pensilvânia. Porém, um dos mais graves episódios acerca dos efeitos deletérios dos poluentes do ar de que se tem notícia ocorreu em Londres durante o inverno de 1952. Em dezembro de 1952 um evento de inversão térmica impediu a dispersão de poluentes, gerados, então, pelas indústrias e pelos aquecedores domiciliares que utilizavam carvão como combustível, e uma nuvem, composta por material particulado e enxofre, em concentrações muito acima do normal, permaneceu sobre a cidade por quatro dias, deixando cerca de quatro mil pessoas mortas durante as duas semanas seguintes devido à poluição do ar. Segundo Logan (1953) o aumento da mortalidade afetou pessoas de todas as idades, mas particularmente aquelas com 45 anos ou mais. As mortes atribuídas a bronquite e a pneumonia aumentaram oito vezes e três vezes, respectivamente, em uma semana. Um aumento considerável no número de mortes ocorreu mesmo no primeiro dia do nevoeiro. Quatro nevoeiros anteriores em Londres, que resultaram em um aumento súbito de mortes, 
foram observados; mas o incidente de 1952 causou de longe o maior aumento. Esses episódios acarretaram em um aumento do número de óbitos em relação à média de óbitos em períodos semelhantes (BRAGA et al. 2005).

Nossos ancestrais já conviviam com a poluição do ar natural, oriunda das erupções vulcânicas e da decomposição da matéria orgânica. Mais tarde, com a expansão da unbanização e da industrialização as atividades antropogências foram intensificadas, o que contribuiu com a aceleração da deterioração da qualidade do ar. Com o crescimento populacional, o desenvolvimento econômico e o crescimento da frota motorizada, as fontes de poluição multiplicaram-se, agravando o problema, mesmo em áreas não industrializadas (LIRA, 2009).

Segundo Holgate et al. (1999), um nível elevado dos poluentes pode ocasionar desde irritação dos olhos, nariz e garganta, bronquite e pneumonia, até doenças respiratórias crônicas, câncer de pulmão, problemas cardíacos, entre outros. Segundo Cançado et al. (2006), isso ocorre,

Pois a poluição do ar causa uma resposta inflamatória no aparelho respiratório induzida pela ação de substâncias oxidantes, as quais acarretam aumento da produção, da acidez, da viscosidade e da consistência do muco produzido pelas vias aéreas, levando, consequentemente, à diminuição da resposta e/ou eficácia do sistema mucociliar. O aumento da poluição do ar tem sido associado ao aumento da viscosidade sanguínea, de marcadores inflamatórios (proteína $\mathrm{C}$ reativa, fibrinogênio) e da progressão de arteriosclerose, a alterações da coagulação, à redução da variabilidade da frequência cardíaca (indicador de risco para arritmia e morte súbita), à vasoconstricção e ao aumento da pressão arterial, todos fatores de risco para doenças cardiovasculares.

Diversos estudos epidemiológicos têm demonstrado associações significativas entre a exposição a concentrações elevadas de poluentes atmosféricos e os problemas de saúde (OSTRO et al., 1996; MARTINS et al., 2002; GOUVEIA et al., 2003; ALMEIDA, 2006; NASCIMENTO et al., 2006; CURTIS, et al., 2006; BRAGA et al., 2007; SOUZA et al., 2014). Para detalhes sobre os principais poluentes, suas fontes e efeitos sobre a saúde humana consultar Arbex et al. (2012). 
Nascimento et al. (2006) investigaram dados diários do número de internações por pneumonia na cidade de São José dos Campos - SP, dados de concentrações médias diárias dos poluentes dióxido de enxofre $\left(\mathrm{SO}_{2}\right)$, ozônio troposférico $\left(\mathrm{O}_{3}\right)$ e material particulado inalável $\left(\mathrm{MP}_{10}\right)$, além de dados de dois parâmetros meteorológicos - temperatura e umidade relativa do ar. Os autores utilizaram modelos aditivos generalizados de regressão de Poisson para estimar a associação entre as internações por pneumonia e a poluição atmosférica. Os três poluentes apresentaram efeitos defasados nas internações por pneumonia, iniciada três a quatro dias após a exposição, e decaindo rapidamente. Na estimativa de efeito acumulado de oito dias, observou-se, ao longo desse período, que para aumentos de $24,7 \mu \mathrm{g}^{-3}$ na concentração de $\mathrm{MP}_{10}$, houve um acréscimo de 9,8 \% nas internações.

Vale dizer que os modelos aditivos generalizados (MAG) são uma extensão dos modelos lineares generalizados. Neste grupo de modelos estatísticos a variável dependente ou resposta (Y) é um processo de contagem e as varáveis independentes são variáveis canditadas a explicar o comportamento da série ao longo do tempo. No MAG cada variável independente analisada não entra no modelo com o seu valor, mas, sim, adotando uma função não paramétrica de forma não especificada, que é estimada a partir de curvas de alisamento. Sendo assim, não é necessário assumir uma relação linear e/ou aditiva entre a variável dependente e a variável independete em estudo. Maiores detalhes desta classe de modelos podem ser vistos nos trabalhos de Latore e Cardoso (2001), Souza et al. (2014), Freitas et al. (2016) e Souza et al. (2018).

Braga et al. (2007) avaliaram os efeitos agudos do $\mathrm{MP}_{10}$ sobre os atendimentos em pronto-socorro por doenças cardiovasculares e respiratórias no Município de Itabira - MG. Os resultados evidenciam que elevações de $10 \mu \mathrm{g} \cdot \mathrm{m}^{-3}$ de $\mathrm{MP}_{10}$ foram associadas aos aumentos nos atendimentos por doenças respiratórias em torno de 4\%, no dia corrente e no 
dia seguinte, para crianças menores de 13 anos, e de $12 \%$ nos três dias subsequentes para os adolescentes entre 13 e 19 anos de idade. Já por doenças cardiovasculares, houve um efeito agudo, principalmente para os indivíduos com idade entre 45 e 64 anos.

Ikefuti, Barrozo e Braga (2018) avaliaram associações entre acidente vascular cerebral (AVC) e temperatura média do ar, usando dados de mortalidade registrados e dados de estações meteorológicas de 2002 a 2011 na cidade de São Paulo. Uma análise de séries temporais foi aplicada a 55.633 casos de mortalidade. De acordo com os resultados apresentados a temperatura média do ar está associada à mortalidade por acidente vascular cerebral na cidade de São Paulo para homens e mulheres. Vale ressaltar que, segundo os autores no Brasil, as doenças crônicas são responsáveis pela maior porcentagem de todas as mortes entre homens e mulheres. Entre as doenças cardiovasculares, o AVC é a principal causa de morte, sendo responsável por $10 \%$ de todas as mortes.

Na Região da Grande Vitória (RGV), Souza et al. (2014) realizaram um estudo cujo objetivo foi investigar a associação entre concentrações dos poluentes atmosféricos e atendimentos diários por causas respiratórias em crianças. Foram analisadas as contagens diárias de admissões hospitalares de crianças menores de 6 anos e as concentrações diárias de poluentes atmosféricos, $\mathrm{MP}_{10}, \mathrm{SO}_{2}$, dióxido de nitrogénio $\left(\mathrm{NO}_{2}\right), \mathrm{O}_{3}$ e monóxido de carbono (CO), de janeiro de 2005 a dezembro de 2010. Os autores combinaram duas técnicas para a análise estatística: modelo de regressão de Poisson em modelos aditivos generalizados e análise de componentes principais. Os resultados mostraram que o aumento de $10.49 \mu \mathrm{g} \mathrm{m}^{-3}$ (intervalo interquartílico) nos níveis do poluente $\mathrm{MP}_{10}$ levou a um aumento de $3,0 \%$ do valor do risco relativo estimado por meio do modelo aditivo generalizado, enquanto no modelo aditivo generalizado usual a estimativa foi de 2,0\%. Ainda, segundo os resultados, existe uma relação significativa, ao nível de 95\% de confiabilidade, entre os níveis de concentração dos 
poluentes e o número de atendimentos hospitalares em crianças menores de 6 anos, mesmo em um ambiente com níveis abaixo dos padrões recomendados pelo Conselho Nacional do Meio Ambiente (CONAMA) e pela World Health Organization (WHO).

Freitas et al. (2016) analisaram o impacto da poluição atmosférica na morbidade respiratória e cardiovascular de crianças e adultos em Vitória, ES. Foi realizado um estudo utilizando modelos de séries temporais via regressão de Poisson a partir de dados de hospitalizações e poluentes em Vitória, de 2001 a 2006. Foram testadas como variáveis independentes $\mathrm{MP}_{10}$, o $\mathrm{SO}_{2}$ e o $\mathrm{O}_{3}$, em defasagem simples e acumulada até cinco dias. Introduziram-se temperatura, umidade e variáveis indicadoras dos dias da semana e feriados da cidade como variáveis de controle nos modelos. Os autores observaram que, para cada incremento de $10 \mu \mathrm{g} \cdot \mathrm{m}^{-3}$ dos poluentes $\mathrm{MP}_{10}, \mathrm{SO}_{2}$ e $\mathrm{O}_{3}$, houve aumentos no risco relativo percentual (RR\%) para as hospitalizações por doenças respiratórias, totais de 9,67; 6,98 e 1,93, respectivamente. De acordo com os resultados da pesquisa, as doenças respiratórias apresentaram relação forte e consistente com os poluentes pesquisados em Vitória. O conjunto de dado utilizado como variáveis dependenetes nos modelos foi cotagens diárias de internações pelas causas invertigadas: doenças respiratórias (CID10:J00-J99) como por exemplo, infecções agudas das vias aéreas superiores, doenças crônicas das vias aéreas inferiores, doenças pulmonares devidas a agentes externos, entre outras e doenças cardiovasculares (CID10: I00-I99) em maiores de 39 anos como, doenças hipertensivas, doenças isquêmicas do coração, doenças das artérias, das arteríolas e dos capilares.

Os efeitos causados ao meio ambiente e à saúde da população por causa da emissão de poluentes atmosféricos podem não ser apenas locais, pois dependem de fatores da região, como o relevo do entorno da fonte de emissão, as condições meteorológicas e a natureza dos poluentes. Isso significa que esses poluentes podem viajar milhares de quilômetros pela 
atmosfera, atingindo, assim, comunidades distantes do ponto de emissão (LEITE, et al., 2011).

Vale frisar que, de acordo com Moreira, Tirabassi e Moraes (2008), as condições meteorológicas desempenham um papel importantíssimo na dispersão ou acumulação de poluentes, uma vez que estas condições atuam através de dois fenômenos fundamentais: o transporte e a difusão. Além disso, as condições meteorológicas influenciam a deposição no solo dos poluentes. Liu e Johnson (2002) descreveram que a poluição do ar está associada, geralmente, a fatores como temperatura, umidade relativa, velocidade e direção do vento, entre outros. Como exemplo, tem-se que a baixa umidade relativa e a reduzida velocidade do vento tendem a elevar os níveis de poluentes. Já a ocorrência de precipitação pluviométrica e o aumento da velocidade do vento contribuem para a dispersão dos poluentes e, consequentemente, para a redução da concentração dos mesmos.

Como grandes projetos industriais foram implantados na RGV desde o início da década de 1970, a indústria era apontada como a principal fonte de poluição da área. De acordo com o IEMA (2013), porém, o crescimento da frota veicular e os empreendimentos imobiliários têm alterado o perfil da região nos últimos anos. O inventário de emissões atmosféricas da Região da Grande Vitória (ECOSOFT, 2011) estimou que os veículos e a indústria minero-siderúrgica foram os principais responsáveis pelas emissões de $\mathrm{CO}$ e hidrocarbonetos, a indústria minero-siderúrgica foi responsável por mais de $70 \%$ da emissão de $\mathrm{SO}_{2}$ para a atmosfera e por mais de $45 \%$ dos óxidos de nitrogênio $\left(\mathrm{NO}_{\mathrm{x}}\right)$, e indicou como principais responsáveis pelos níveis de $\mathrm{MP}_{10}$ a ressuspensão do solo (69,3\%), as indústrias (19,6\%) e os veículos (Escapamento e Evaporativa) (3,9\%). Com o aumento da poluição do ar na região, aumentou também a preocupação com os efeitos adversos à saúde humana, à fauna e à flora. Dessa forma, este trabalho objetivou avaliar os impactos das variáveis meteorológicas temperatura, a umidade relativa, a velocidade do vento e a precipitação na probabilidade de ocorrência de 
picos/episódios de concentração de poluentes na RGV, por meio do modelo Logit.

O modelo Logit é uma ferramenta estatística que permite o ajuste de um conjunto de variáveis independentes a uma variável dependente dicotômica, ou seja, é possível estimar probabilidades de ocorrências em variáveis dependentes do tipo binário. O modelo Logit aplicado na modelagem de dados ambientais tem sido utilizado por Kuchenho e Thamerus (1996), Mendes e Vega (2011), Silva et al. (2011), Gehring et al. (2013), entre outros autores. Com objetivo semelhante, cabe ressaltar o trabalho de Leite et al. (2011), que analisaram a qualidade do ar atmosférico de Uberlândia, em Minas Gerais, por meio de modelos de regressão logística simples. Os autores utilizaram dados do período de 2003 a 2008 de concentrações de $\mathrm{MP}_{10}$ e de variáveis meteorológicas. De acordo com os resultados, os modelos logísticos simples mostraram que as variáveis meteorológicas precipitação e umidade relativa contribuem significativamente para a redução das concentrações de $\mathrm{MP}_{10}$.

\section{Materiais e métodos}

\section{Área de estudo e apresentação das variáveis}

Para a realização deste estudo, utilizaram-se séries temporais de concentrações de poluentes atmosféricos e de variáveis meteorológicas monitorados na RGV (conforme apresentado na Tabela 1),_Espírito Santo, Brasil, região que é constituída pelos municípios de Vitória, Vila Velha, Cariacica, Serra e Viana. Segundo o Instituto Brasileiro de Geografia e Estatística (IBGE, 2010), a RGV abrange uma área de $1.461 \mathrm{Km}^{2}$, com aproximadamente 1.475.332 habitantes, sendo um dos principais polos de desenvolvimento urbano e industrial do Estado. A região sofre com diversos tipos de problemas ambientais, entre os quais está a deterioração da qualidade do ar, devido às emissões atmosféricas por indústrias, pela frota 
veicular e pela ressuspensão do solo causada pelo vento e pelo tráfego veicular.

Vale ressaltar que a RGV possui uma Rede Automática de Monitoramento da Qualidade do Ar (RAMQAR) inaugurada em julho de 2000, de propriedade do IEMA. Essa rede é distribuída em oito estações, a saber: no município da Serra, duas estações localizadas nas regiões de Laranjeiras e de Carapina; no município de Vitória, três estações localizadas nas regiões de Jardim Camburi, Enseada do Suá e Centro de Vitória; no município de Vila Velha, duas estações localizadas nas regiões do Ibes e Centro de Vila Velha; por fim, no município de Cariacica, uma estação em Cariacica. A localização espacial das estações de monitoramento da RAMQAR está ilustrada na Figura 1.

A RAMQAR monitora os seguintes poluentes: Partículas Totais em Suspensão (PTS), $\mathrm{MP}_{10}, \mathrm{O}_{3}, \mathrm{NO}_{\mathrm{x}}, \mathrm{CO}$ e Hidrocarbonetos (HC). Ainda, realiza o monitoramento dos seguintes parâmetros meteorológicos: Direção dos Ventos (DV), Velocidade dos Ventos (VV), Precipitação Pluviométrica (PP), Umidade Relativa do Ar (UR), Temperatura (T), Pressão Atmosférica (P) e Radiação Solar (I). Na análise deste trabalho, as variáveis PTS, $\mathrm{MP}_{10}, \mathrm{SO}_{2}$, $\mathrm{CO}, \mathrm{NO}_{2}, \mathrm{O}_{3}$, velocidade do vento, umidade relativa, precipitação e temperatura foram utilizadas conforme descrição da Tabela 2. 
Figura 1: Localização espacial das estações de monitoramento da qualidade do ar da RGV.

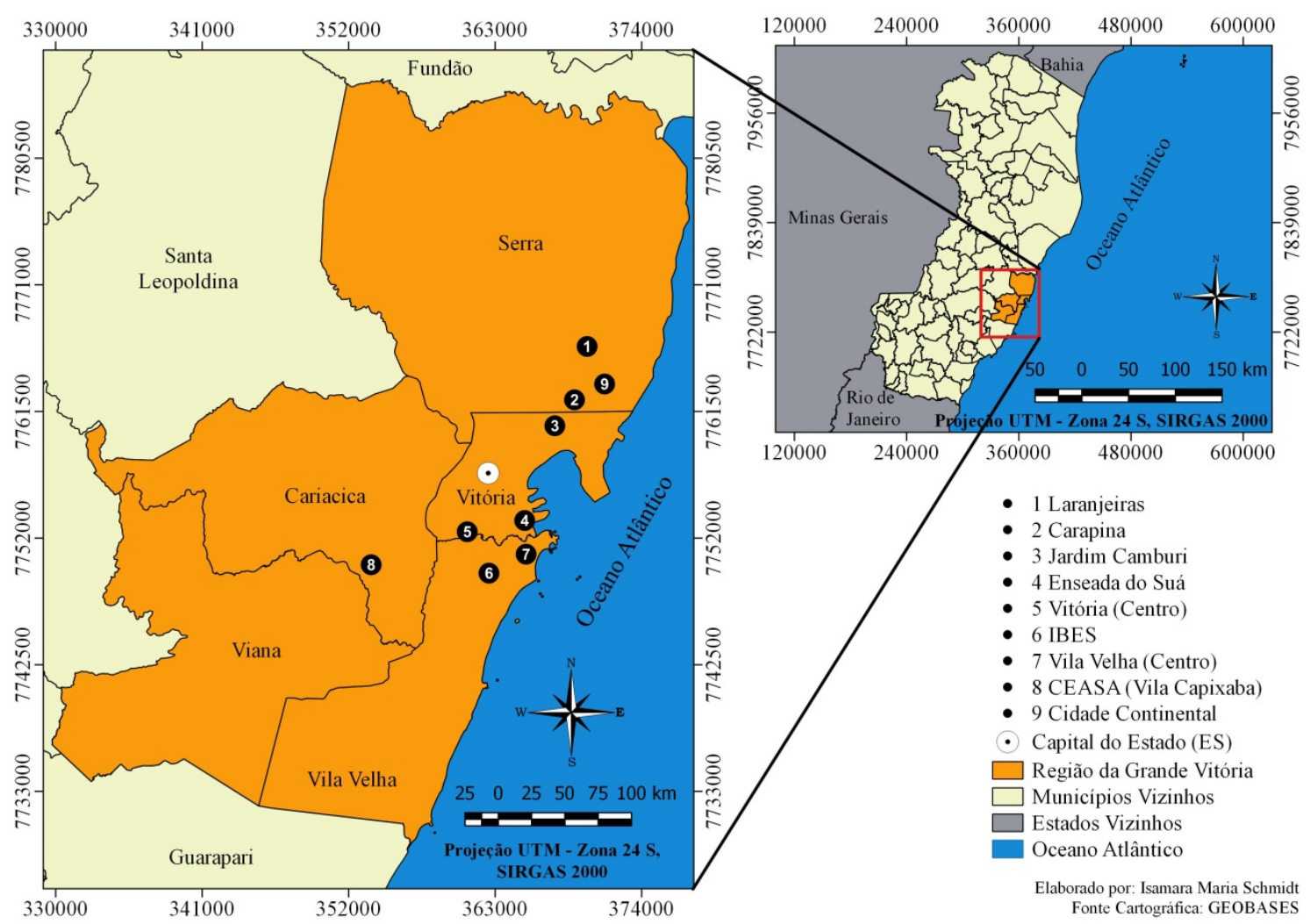

Fonte: Elaborado para os autores por Isamara Maria Schmidt, 2018.

Tabela 1: Parâmetros meteorológicos e poluentes monitorados em cada estação RAMQAR

\begin{tabular}{|c|c|c|c|c|c|c|c|c|}
\hline Estação & PTS & $\mathrm{PM}_{10}$ & $\mathrm{SO}_{2}$ & $\mathrm{CO}$ & $\mathrm{NO}_{\mathrm{x}}$ & $\mathrm{HC}$ & $\mathrm{O}_{3}$ & Meteorologia \\
\hline Laranjeiras & $\mathrm{X}$ & $\mathrm{X}$ & $\mathrm{X}$ & $\mathrm{X}$ & $\mathrm{X}$ & & $\mathrm{X}$ & \\
\hline Carapina & $\mathrm{X}$ & $\mathrm{X}$ & & & & & & DV, VV, UR, PP, P, T, I \\
\hline Jardim & $\mathrm{X}$ & $\mathrm{X}$ & $\mathrm{X}$ & & $\mathrm{X}$ & & & \\
\hline $\begin{array}{l}\text { Camburi } \\
\text { Enseada do } \\
\text { Suá }\end{array}$ & $\mathrm{X}$ & $\mathrm{X}$ & $\mathrm{X}$ & $\mathrm{X}$ & $\mathrm{X}$ & $\mathrm{X}$ & $\mathrm{X}$ & $\mathrm{DV}, \mathrm{VV}$ \\
\hline Vitória Centro & $\mathrm{X}$ & $\mathrm{X}$ & $\mathrm{X}$ & $\mathrm{X}$ & $\mathrm{X}$ & $\mathrm{X}$ & & \\
\hline Ibes & $\mathrm{X}$ & $\mathrm{X}$ & $\mathrm{X}$ & $\mathrm{X}$ & $\mathrm{X}$ & $\mathrm{X}$ & $\mathrm{X}$ & $\mathrm{DV}, \mathrm{VV}$ \\
\hline Vila Velha & $\mathrm{y}$ & $\begin{array}{l}\mathrm{X} \\
\mathrm{Y}\end{array}$ & $\mathrm{X}$ & & $\mathrm{Y}$ & & $\mathrm{y}$ & DV VY UB T \\
\hline
\end{tabular}

Fonte: Adaptado de Relatório da Qualidade do Ar da Região da Grande Vitória, 2013. 
Tabela 2: Descrição das variáveis $\mathrm{PTS}, \mathrm{MP}_{10}, \mathrm{SO}_{2}, \mathrm{CO}, \mathrm{NO}_{2}, \mathrm{O}_{3}$, velocidade do vento, umidade, precipitação e temperatura

\begin{tabular}{|c|c|c|}
\hline Variáveis & Unidades & Descrição \\
\hline $\begin{array}{l}\mathrm{PTS}, \quad \mathrm{MP}_{10} \\
\mathrm{SO}_{2}, \mathrm{CO}, \mathrm{NO}_{2} \\
\mathrm{e} \mathrm{O}_{3}\end{array}$ & & $\begin{array}{l}\text { Valor máximo medido entre as estações que fazem o } \\
\text { monitoramento do poluente, para cada dia da amostra de } \\
\text { dados. }\end{array}$ \\
\hline $\begin{array}{l}\text { Velocidade do } \\
\text { Vento }\end{array}$ & $m \cdot s^{-1}$ & $\begin{array}{l}\text { Valor máximo medido entre as estações de Carapina, } \\
\text { Enseada do Suá, Ibes e Cariacica. }\end{array}$ \\
\hline $\begin{array}{l}\text { Umidade } \\
\text { relativa }\end{array}$ & $\%$ & $\begin{array}{l}\text { Como existem muitos dados faltantes para a estação de } \\
\text { Cariacica, adotou-se o valor de Carapina. }\end{array}$ \\
\hline Precipitação & $\mathrm{mm}$ & $\begin{array}{l}\text { Valores medidos na estação de Carapina, única que possui } \\
\text { medição para tal variável. }\end{array}$ \\
\hline Temperatura & ${ }^{\circ} \mathrm{C}$ & $\begin{array}{l}\text { Média aritmética entre as estações de Carapina e Cariacica, } \\
\text { únicas que possuem medições para tal variável. }\end{array}$ \\
\hline
\end{tabular}

Fonte: Org. dos autores, 2017.

O IEMA classifica diariamente a qualidade do ar da RGV como "boa" e "não boa". Nesse último caso, são englobadas as classificações "regular", "inadequada", "má", "péssima" e "crítica". A qualidade é considerada "boa" se a concentração de cada poluente for igual ou inferior aos valores apresentados na Tabela 3. Já a classificação de "não boa” ocorre quando a concentração é superior aos valores apresentados na Tabela 3.

Este trabalho preocupou-se com os picos de concentração. As concentrações de cada poluente foram representadas pelo valor máximo medido dentre as estações que fazem o monitoramento desses poluentes (Tabela 1), para cada dia da amostra de dados. No mais, como a regressão logística considera a variável dependente como dicotômica, a concentração de cada poluente foi transformada em uma variável dummy, apresentando a seguinte classificação: um (1) para classificação "não boa" (picos de concentração) e zero (0) para "boa".

Tabela 3: Limites de concentração para classificação da qualidade do ar como "boa" e "não boa"

\begin{tabular}{lllllll}
\hline & PTS & $\mathrm{PM}_{10}$ & $\mathrm{SO}_{2}$ & $\mathrm{NO}_{2}$ & $\mathrm{O} 3$ & $\mathrm{CO}$ \\
Classificação & $\begin{array}{l}\mu \mathrm{g} / \mathrm{m}^{3} \\
\text { Média } 24 \mathrm{~h}\end{array}$ & $\begin{array}{l}\mu \mathrm{g} / \mathrm{m}^{3} \\
\text { Média } 24 \mathrm{~h}\end{array}$ & $\begin{array}{l}\mu \mathrm{g} / \mathrm{m}^{3} \\
\text { Média } 24 \mathrm{~h}\end{array}$ & $\begin{array}{l}\mu \mathrm{g} / \mathrm{m}^{3} \\
\text { Média } 1 \mathrm{~h}\end{array}$ & $\begin{array}{l}\mu \mathrm{g} / \mathrm{m}^{3} \\
\text { Média } 8 \mathrm{~h}\end{array}$ & $\begin{array}{l}\mu \mathrm{g} / \mathrm{m}^{3} \\
\text { Média } 1 \mathrm{~h}\end{array}$ \\
\hline Boa & $0-65$ & $0-45$ & $0-40$ & $0-50$ & $0-70$ & $0-5.000$ \\
Não boa & $>66$ & $>46$ & $>41$ & $>51$ & $>71$ & $>5.001$ \\
\hline
\end{tabular}

Fonte: IEMA, 2017. 


\section{Modelo Logit}

Para verificar a influência das variáveis meteorológicas (variáveis preditoras) temperatura, umidade relativa, velocidade do vento $\mathrm{e}$ precipitação pluviométrica na probabilidade de ocorrência de picos de concentração de poluentes atmosféricos (classificação "não boa" na qualidade do ar) na RGV, foi utilizado o modelo Logit (GUJARATI; PORTER 2008), que admite valores discretos, zero e um (variável binária) para a variável dependente. Um dos principais objetivos dos modelos de resposta binária é calcular a probabilidade de um dado evento, com determinado conjunto de atributos, de fato acontece (MONTE, ALBUQUERQUE e REISEN, 2016).

No modelo Logit utiliza-se uma função de distribuição acumulada logística, dada por

$$
L\left(\mathrm{X}_{\mathrm{t}} \boldsymbol{\beta}\right)=\frac{1}{1+e^{-\left(\beta_{0}+\sum \sum_{=1}^{\mathrm{k}}\left(\beta_{1} X_{t 1}\right)^{2}\right.}}
$$

em que L representa a função de distribuição logística; $\mathrm{X}_{\mathrm{t}}$,variáveis independentes; $\boldsymbol{\beta}=\left\{\beta_{0}, \beta_{1}, \cdots, \beta_{\mathrm{k}}\right\}$, vetor de parâmetros a serem estimados; $\mathbf{k}$, o número de variáveis explicativas; $e$, base do logaritmo natural; e, $\mathrm{t}=1_{\imath} \cdots, \mathrm{n}$ (número de observações).

A ocorrência ou não de uma classificação "não boa" da qualidade do ar depende de vários fatores. Como os parâmetros dessa ocorrência não são observáveis para cada ponto do tempo t, pode-se definir uma variável latente ou não observada, $Y_{t}^{*}$, como

$$
\mathrm{Y}_{\mathrm{t}}^{*}=\beta_{0}+\sum_{\mathrm{i}=1}^{\mathrm{k}} \beta_{1} \mathrm{X}_{\mathrm{t} 1}+\mu_{\mathrm{t}}
$$

em que $\mathrm{Y}_{\mathrm{t}}^{*}$ é variável latente e $\mu_{\mathrm{t}}$, erro aleatório. Para detalhes ver Gujarati e Porter (2008). 
A ocorrência de uma determinada classificação pode ser descrita pela variável binária $Y_{t}$, tal que $Y_{t}=1$, se a classificação é “não boa”, e $Y_{t}=0$, se é "boa". Esses valores observados de $\mathrm{Y}_{\mathrm{t}}$ estão relacionados com $\mathrm{Y}_{\mathrm{t}}^{*}$, como segue:

$$
\begin{aligned}
& Y_{t}=1, \text { se } Y_{t}^{*}>0 ; \text { e, } Y_{t}^{*}=0, \text { se } Y_{t}^{*}=0, \\
& \operatorname{Prob}\left(Y_{t}=1\right)=\operatorname{Prob}\left(Y_{t}^{*}>0\right)=\operatorname{Prob}\left(\mu_{t}>-\left(\beta_{0}+\sum_{t=1}^{k} \beta_{1} X_{t 1}\right)\right), \\
& \operatorname{Prob}\left(Y_{t}=0\right)=\operatorname{Prob}\left(Y_{t}^{*}=0\right)=\operatorname{Prob}\left(\mu_{t} \leq-\left(\beta_{0}+\sum_{t=1}^{k} \beta_{1} X_{t 1}\right)\right) .
\end{aligned}
$$

Os parâmetros do modelo são estimados pelo Método de Máxima Verossimilhança. A probabilidade de ocorrência da classificação "não boa" (a) e a probabilidade de ocorrência da classificação "boa" (b) pode ser calculada pelas seguintes expressões:

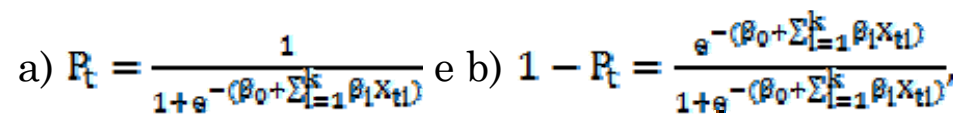

sendo ${ }^{\mathrm{P}_{\mathrm{t}}}$ igual a probabilidade de ocorrência da classificação "não boa", $\mathrm{e}^{1-\mathrm{P}_{\mathrm{t}}}$, probabilidade de ocorrência da classificação "boa".

Para determinar o efeito marginal de cada variável preditora, sobre a probabilidade de ocorrência da classificação "não boa”, é necessário usar os valores médios das variáveis explicativas. $\mathrm{O}$ efeito marginal da variável $\mathrm{X}_{\mathrm{t} 1}$ sobre a variável dependente é descrito pela expressão

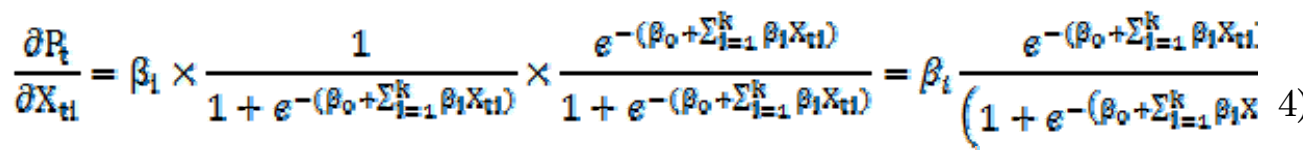

A Equação 4 mostra o efeito marginal sobre $P_{t}$, de um aumento em $\mathrm{X}_{\mathrm{t1}}$. Observa-se que o efeito marginal de cada variável explicativa sobre a probabilidade não é constante, visto que depende do valor médio de cada 
variável $\mathrm{X}_{\mathrm{t1}}$. Para detalhes, ver em Gujarati e Porter (2008) e Hill, Judge e Griffiths (2010).

\section{Resultados e discussão}

Para um entendimento preliminar das variáveis em estudo, são apresentadas algumas medidas descritivas (Tabelas 4 e 5). Para detalhes sobre as medidas apresentadas e suas formulações matemáticas, consultar em Gujarati e Porter (2008) e Morettin e Bussab (2017).A análise corresponde às variáveis apresentadas na Tabela 2, para o período de 01/01/2012 a 31/12/2014, perfazendo um total de 1096 observações. Todas as análises foram feitas utilizando o software livre $\mathrm{R}$ (R DEVELOPMENT CORE TEAM, 2016). A presença de dados faltantes nas séries motivou o uso da metodologia de imputação via algoritmo EM (expectation-maximisation), proposta por Junger e Leon (2015) e implementada na biblioteca R mtsdi (multivariante time-series data imputation).

Tabela 4: Estatísticas descritivas das variáveis poluentes atmosféricos

\begin{tabular}{lcccccc}
\hline \multirow{2}{*}{ Estatísticas } & \multicolumn{7}{c}{ Variáveis } \\
\cline { 2 - 7 } & $\mathrm{MP}_{10}$ & $\mathrm{PTS}$ & $\mathrm{SO} 2$ & $\mathrm{NO}_{2}$ & $\mathrm{CO}$ & $\mathrm{O}_{3}$ \\
\hline Média & 48,3911 & 87,1433 & 21,2563 & 74,3990 & 844,5010 & 35,7936 \\
Mediana & 46,3750 & 79,5833 & 19,7050 & 70,1688 & 805,4279 & 35,5504 \\
Desvio padrão & 16,9144 & 36,7769 & 9,9869 & 26,8003 & 285,4049 & 9,1888 \\
Coeficiente de & & & & & & \\
variação & 34,9535 & 42,2028 & 46,9832 & 36,0224 & 33,7957 & 25,6717 \\
Valor máximo & 125,0000 & 279,1667 & 73,1713 & 197,2292 & 1893,5408 & 67,1804 \\
Valor mínimo & 12,2500 & 15,0000 & 2,4221 & 19,8208 & 232,7021 & 0,0000 \\
\hline
\end{tabular}

Fonte: Org. dos autores, 2017.

Nota-se que (Tabela 4), em média, as concentrações de $\mathrm{MP}_{10}$, PTS e $\mathrm{NO}_{2}$ ultrapassaram os valores apresentados na Tabela 3 para a classificação de qualidade do ar "boa", já as concentrações de $\mathrm{SO}_{2}, \mathrm{O}_{3}$ e $\mathrm{CO}$ não ultrapassaram. Porém, o desvio padrão e o coeficiente de variação alto 
indicam uma média pouco representativa e uma grande variabilidade dos dados. Por exemplo, os resultados mostram que a concentração máxima de PTS foi mais do que o triplo do valor médio, demostrando a grande variabilidade desse poluente na RGV.

A Tabela 5 apresenta as estatísticas descritivas para as variáveis meteorológicas. Em geral, observando-se os desvios padrão, o coeficiente de variação e as diferenças entre os máximos e mínimos, percebe-se que as variáveis meteorológicas apresentaram grande variabilidade em termos estatísticos, exceção feita à temperatura. Segundo o IEMA (2013), não há muita variabilidade climatológica na RGV.

Tabela 5: Estatísticas descritivas das variáveis meteorológicas

\begin{tabular}{lllll}
\hline Estatísticas & \multicolumn{3}{c}{ Variáveis } \\
\cline { 2 - 5 } & VV & PP & UR & T \\
\hline Média & 2,4256 & 0,1289 & 77,5826 & 25,0952 \\
Mediana & 2,3087 & 0,0000 & 77,0229 & 25,0392 \\
Desvio padrão & 0,7904 & 0,5421 & 6,0259 & 2,4129 \\
Coeficiente de variação & 32,5846 & 420,5203 & 7,7671 & 9,6150 \\
Valor máximo & 5,5412 & 12,7750 & 98,2983 & 31,4000 \\
Valor mínimo & 1,0392 & 0,0000 & 56,8638 & 18,1758 \\
\hline
\end{tabular}

Fonte: Org. dos autores, 2017.

A Tabela 6 mostra as correlações calculadas entre as concentrações de médias diárias de $\mathrm{PTS}, \mathrm{MP}_{10}, \mathrm{SO}_{2}, \mathrm{CO}, \mathrm{NO}_{2}, \mathrm{O}_{3}$, de velocidade do vento, da umidade, de precipitação e de temperatura. Observa-se que as variáveis meteorológicas apresentam relação linear com as concentrações de poluentes. Conforme esperado, os índices de concentrações máximas na RGV estão associados às mudanças dessas variáveis, ou seja, infere-se dos resultados (Tabela 6) que o aumento da velocidade do vento acarreta aumento nas concentrações de $\mathrm{MP}_{10}, \mathrm{SO}_{2}$ e $\mathrm{O}_{3}$ e diminuição das concentrações de PTS, $\mathrm{NO}_{2}$ e $\mathrm{CO}$; o aumento da precipitação acarreta uma diminuição nas concentrações de $\mathrm{MP}_{10}$, PTS e $\mathrm{SO}_{2}$; o aumento da umidade implica em um aumento de $\mathrm{NO}_{2}$ e $\mathrm{CO}$ e em uma diminuição das concentrações de $\mathrm{MP}_{10}$, PTS e $\mathrm{SO}_{2}$. Já o aumento da temperatura acarreta 
um aumento nas contrações de $\mathrm{MP}_{10}$, PTS, $\mathrm{SO}_{2}$ e $\mathrm{O}_{3}$ e diminuição de $\mathrm{NO}_{2} \mathrm{e}$ CO.

Tabela 6: Matriz de correlação entre as variáveis sob estudo

\begin{tabular}{lcccccccccc}
\hline & $\mathrm{MP}_{10}$ & $\mathrm{PTS}$ & $\mathrm{SO} 2$ & $\mathrm{NO}_{2}$ & $\mathrm{CO}$ & $\mathrm{O}_{3}$ & $\mathrm{VV}$ & $\mathrm{PP}$ & $\mathrm{UR}$ & $\mathrm{T}$ \\
\hline $\mathrm{MP}_{10}$ & 1,0000 & & & & & & & & & \\
$\mathrm{PTS}$ & 0,6453 & 1,0000 & & & & & & & \\
$\mathrm{SO} 2$ & 0,2947 & 0,1960 & 1,0000 & & & & & & \\
$\mathrm{NO}_{2}$ & 0,2101 & 0,4105 & $-0,1060$ & 1,0000 & & & & & \\
$\mathrm{CO}$ & 0,3488 & 0,4688 & 0,1825 & 0,6742 & 1,0000 & & & & \\
$\mathrm{O}_{3}$ & 0,0769 & 0,0755 & 0,1697 & $-0,3567$ & $-0,3248$ & 1,0000 & & & & \\
VV & 0,1823 & $-0,0645$ & 0,3023 & $-0,5862$ & $-0,3797$ & 0,3739 & 1,0000 & & & \\
$\mathrm{PP}$ & $-0,1801$ & $-0,1631$ & $-0,0539$ & 0,0098 & 0,0385 & 0,0019 & $-0,0535$ & 1,0000 & & \\
$\mathrm{UR}$ & $-0,1772$ & $-0,0755$ & $-0,0843$ & 0,2178 & 0,3107 & $-0,2146$ & $-0,3671$ & 0,3240 & 1,0000 & \\
$\mathrm{~T}$ & 0,0906 & 0,0966 & 0,4326 & $-0,3405$ & $-0,0786$ & 0,0256 & 0,3222 & $-0,1425$ & $-0,3406$ & 1,0000 \\
\hline
\end{tabular}

Fonte: Org. dos autores, 2017.

A Figura 2 apresenta as séries temporais das concentrações de poluentes atmosféricos, e a Figura 3 as das variáveis meteorológicas no período de análise desse estudo. De um total de 1096 observações (dias) para o poluente $\mathrm{MP}_{10}$, em cerca de $51 \%$ dos dias (médias de 24 horas) a qualidade do ar foi classificação como "não boa"; para o PTS, aproximadamente em $68 \%$ dos dias (médias de 24 horas); para o $\mathrm{SO}_{2}$, a porcentagem foi de $95 \%$ dos dias (médias de 24 horas); para o $\mathrm{NO}_{2}, 64 \%$ das médias horárias foram classificadas como "não boas"; para o $\mathrm{O}_{3}, 0,63 \%$ das médias de oito horas foram classificadas como "não boas". Já para o poluente $\mathrm{CO}$, a qualidade do ar foi classificada como "não boa" uma única vez. 
Figura 2: Séries temporais das concentrações dos poluentes atmosféricos em estudo.
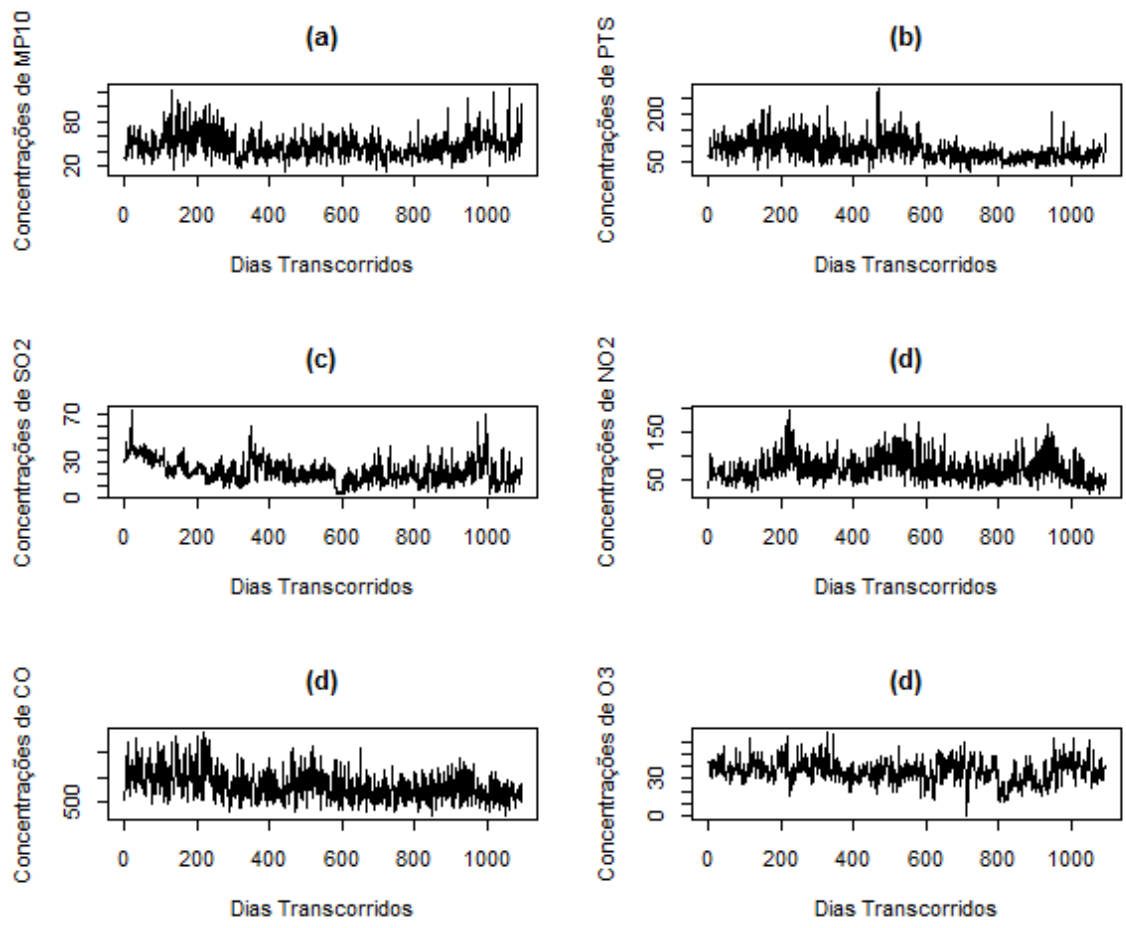

Fonte: Org. dos autores, 2017. 
Figura 3: Séries temporais das variáveis meteorológicas em estudo.

(a)

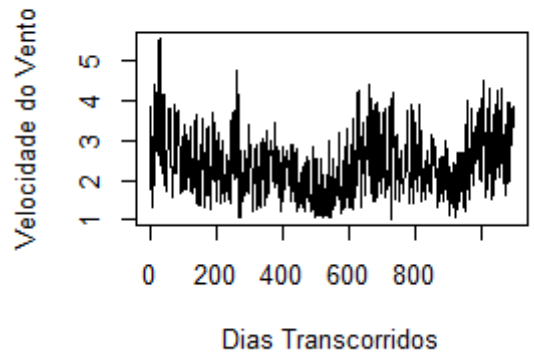

(c)

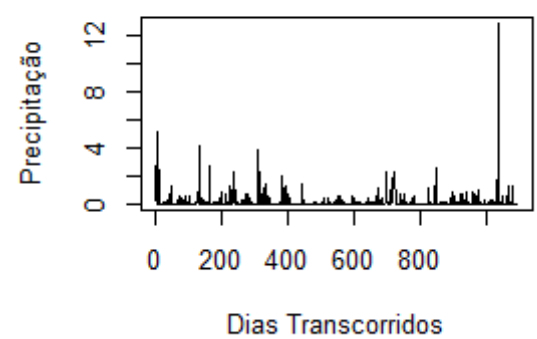

(b)

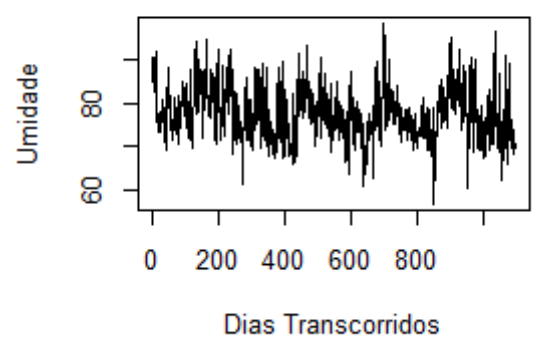

(d)

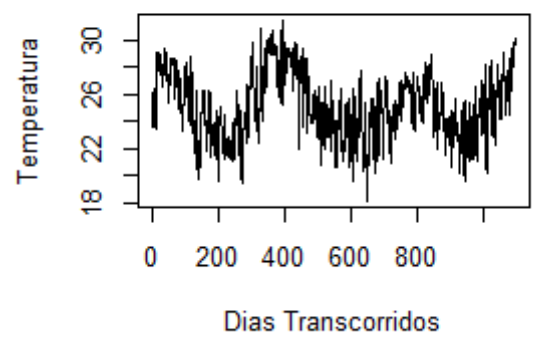

Fonte: Org. dos autores, 2017.

Na Tabela 7 são apresentados os resultados para a regressão logística estimada para cada poluente, considerando como variáveis explicativas a umidade relativa, a velocidade do vento, a precipitação e a temperatura. Também são apresentados os respectivos efeitos marginais de cada variável sobre a probabilidade de ocorrência de qualidade do ar "não boa" para todos os poluentes estudados. Verificou-se que as regressões como um todo foram estatisticamente significativas. Uma vez que para o poluente CO a qualidade do ar foi classificada como "não boa" uma única vez, não foi estimada uma regressão para ele, pois o efeito das variáveis meteorológicas não seria estatisticamente significativo.

Para o Poluente $\mathrm{MP}_{10}$, os resultados mostraram que as variáveis explicativas velocidade do vento, precipitação e umidade interferem significantemente na qualidade do ar "não boa". Como a variável temperatura não foi estatisticamente significativa, os resultados para ela 
não se encontram na Tabela 6. Analisando os efeitos marginais (Tabela 6), verifica-se que, para cada unidade de acréscimo na velocidade do vento na região, ocorreu um aumento de 5,9 pontos percentuais na probabilidade de qualidade do ar "não boa"; para a precipitação um aumento de uma unidade, ocasionou uma redução na probabilidade de qualidade do ar "não boa" de 27,11 pontos percentuais. Já para a variável umidade, o efeito marginal foi muito pequeno.

É importante ressalvar que, para o poluente $\mathrm{MP}_{10}$, os sinais estimados são coerentes com o esperado. O sinal positivo do coeficiente estimado da variável velocidade do vento indica a existência de uma relação direta entre a concentração de $\mathrm{MP}_{10}$ e essa variável, demostrando que o aumento da velocidade do vento eleva a concentração de $\mathrm{MP}_{10}$. Segundo o Relatório da Qualidade do Ar da Grande Vitória (IEMA, 2013), 69,3\% das emissões de $\mathrm{MP}_{10}$ para a atmosfera da RGV estão ligadas à ressuspensão causada pelo vento e pelo tráfego veicular, corroborando com o resultado encontrado, uma vez que o aumento da velocidade do vento tende à elevar a ressuspensão do solo. Já a precipitação pluvial e a umidade apresentaram relação negativa com a concentração de $\mathrm{MP}_{10}$. $\mathrm{O}$ coeficiente negativo no modelo se deve ao processo de deposição úmida e atenuação da ressuspensão do solo (LYRA; ODA-SOUZA; VIOLA, 2011). Durante eventos de precipitação ocorre diminuição do $\mathrm{MP}_{10}$, pois o solo úmido dificulta a ressuspensão do particulado no solo (VARDOULAKIS; KASSOMENOS, 2008).

Para o poluente PTS, os resultados mostraram que a variável precipitação interfere significantemente na qualidade do ar "não boa". Analisando os efeitos marginais (Tabela 7), verifica-se que para cada unidade de acréscimo na precipitação ocasionou uma redução na probabilidade de qualidade do ar "não boa" de 15,69 pontos percentuais; para a temperatura, um aumento de uma unidade ocasionou um aumento na probabilidade de qualidade do ar "não boa" de 2,83 pontos percentuais. 
Como as variáveis velocidade do vento e umidade não foram estatisticamente significativas, os resultados para as mesmas não se encontram na Tabela 7.

Os resultados mostraram que as variáveis meteorológicas velocidade do vento e precipitação interferem nas concentrações de $\mathrm{SO}_{2}$ e $\mathrm{NO}_{2}$ presentes no ar da RGV, favorecendo na redução da poluição atmosférica, como mostra a Tabela 6. Observou-se que um aumento de uma unidade na velocidade do vento acarreta uma redução de 1,16 pontos percentuais na probabilidade de ocorrência de qualidade do ar "não boa" para o poluente $\mathrm{SO}_{2}$ e de 7,79 pontos percentuais para o poluente $\mathrm{NO}_{2}$. Já para a precipitação, um aumento de uma unidade implica em uma redução de 3,95 pontos percentuais na probabilidade de ocorrência de qualidade do ar "não boa" para o poluente $\mathrm{SO}_{2}$. Ressalta-se, ainda, que o efeito marginal da umidade foi muito pequeno, tanto para o $\mathrm{SO}_{2}$ quanto para o $\mathrm{NO}_{2}$; da precipitação e da temperatura foi pequeno para o $\mathrm{NO}_{2}$. Os resultados revelaram, também, que as variações das variáveis meteorológicas não são estatisticamente significantes para explicar as concentrações de $\mathrm{O}_{3}$ na $\mathrm{RGV}$.

Vale ressaltar que, as condições meteorológicas influenciam as condições de mistura vertical do ar, e as concentrações de $\mathrm{O}_{3}$ aumentam, conforme diminuem as condições de mistura. Porém, sabe-se que as concentrações de $\mathrm{NO}_{\mathrm{x}}$ aumentam conforme aumeta a estabilidade vertical da atmosfera, o que afeta diretamente as concentrações de $\mathrm{O}_{3}$. Além disso, as concentrações de ozônio troposférico num determinado local são afetadas pela constituição atmosférica, e esta é notadamente modificada em grandes centros urbanos onde há grandes emissões de precursores devido às atividades antrópicas (CHIQUETTO, 2008).

Para corroborar com os resultados apresentados na Tabela 7, é válido ressaltar que os sinais dos coeficientes estimados para as variáveis meteorológicas estão de acordo com o esperado. O sinal negativo dos coeficientes estimados das variáveis velocidade do vento e precipitação 
indica a existência de uma relação inversa entre as concentrações de $\mathrm{SO}_{2} \mathrm{e}$ essas variáveis, demostrando que o aumento da velocidade do vento e da precipitação diminuem as concentrações de $\mathrm{SO}_{2}$. Segundo Derisio (2012), a poluição numa região ocorre em função das atividades da comunidade aí presentes e das condições meteorológicas. A ocorrência de precipitação indica instabilidade atmosférica, ou seja, favorece a dispersão de poluentes pelos movimentos de ar e as gotas de chuva também removem quantidades consideráveis desses poluentes. O aumento da velocidade do vento favorece a dispersão do $\mathrm{SO}_{2}$ e do $\mathrm{NO}_{2}$ na atmosfera. Para Derisio (2012), o vento é o primeiro mecanismo atmosférico de transporte de contaminantes.

Tabela 7: Equações logísticas considerando as variáveis explicativas e seus efeitos marginais para cada poluente

\begin{tabular}{|c|c|c|c|c|c|c|}
\hline Modelo & Variáveis & Coeficientes & $\begin{array}{l}\text { Erro- } \\
\text { padrão }\end{array}$ & Valor de $\mathrm{Z}$ & Valor-p & $\begin{array}{l}\text { Efeito } \\
\text { marginal }\end{array}$ \\
\hline \multirow[t]{7}{*}{ ModeloPM $_{10}$} & Constante & 0,8654 & 1,0603 & 0,8162 & 0,4144 & - \\
\hline & VV & $0,3771^{*}$ & 0,0880 & 4,2863 & 0,0000 & 0,0598 \\
\hline & PREC & $-1,7099^{*}$ & 0,3815 & $-4,4819$ & 0,0000 & $-0,2711$ \\
\hline & UMID & $-0,0204$ & 0,0126 & $-1,6130$ & 0,1067 & $-0,0032$ \\
\hline & TEMP & & & - & & \\
\hline & Constante & $-2,4256$ & 0,7212 & $-3,3631$ & 0,0008 & - \\
\hline & VV & - & - & - & - & - \\
\hline \multirow[t]{5}{*}{ ModeloPTS } & PREC & $-0,7391^{*}$ & 0,1966 & $-3,7585$ & 0,0002 & $-0,1569$ \\
\hline & UMID & - & & - & - & \\
\hline & TEMP & $0,1331^{*}$ & 0,0288 & 4,6238 & 0,0000 & 0,0283 \\
\hline & Constante & $-0,4062$ & 1,4096 & $-0,2882$ & 0,7732 & - \\
\hline & VV & $-0,1854^{* *}$ & 0,0937 & $-1,9774$ & 0,0480 & $-0,0116$ \\
\hline \multirow[t]{5}{*}{ ModeloSO $_{2}$} & PREC & $-0,6310^{*}$ & 0,2087 & $-3,0242$ & 0,0025 & $-0,0395$ \\
\hline & UMID & $-0,0221 * * *$ & 0,0131 & $-1,6907$ & 0,0909 & $-0,0014$ \\
\hline & TEMP & $0,1387^{*}$ & 0,0310 & 4,4748 & 0,0000 & 0,0087 \\
\hline & Constante & 3,0386 & 0,2321 & 13,0904 & 0,0000 & - \\
\hline & VV & $-0,3185^{*}$ & 0,0136 & $-23,4487$ & 0,0000 & $-0,0779$ \\
\hline \multirow[t]{5}{*}{ ModeloNO $_{2}$} & PREC & $0,0363^{*}$ & 0,0122 & 2,9869 & 0,0028 & 0,0089 \\
\hline & UMID & $-0,0332^{*}$ & 0,0017 & $-19,9910$ & 0,0000 & $-0,0081$ \\
\hline & TEMP & $0,0373^{*}$ & 0,0052 & 7,2506 & 0,0000 & 0,0091 \\
\hline & Constante & 0,5211 & 1,2799 & 0,4071 & 0,6839 & - \\
\hline & VV & - & - & - & - & - \\
\hline \multirow[t]{3}{*}{$\mathrm{ModeloO}_{3}$} & PREC & - & - & - & - & - \\
\hline & UMID & $-0,0770 *$ & 0,0188 & $-4,0875$ & 0,0000 & $-0,0003$ \\
\hline & TEMP & & & - & - & \\
\hline
\end{tabular}

Nota: 1) *Significativo a $1 \%$, **Significativo a $5 \%$, ***Significativo a $10 \%$; e, 2) As estimativas foram realizadas utilizando o método de covariâncias robusta GLM (Modelo Linear Generalizado).

Fonte: Org. dos autores, 2017. 
Referente ao coeficiente da variável temperatura, para o poluente $\mathrm{SO}_{2}$, embora pequeno, ele foi estatisticamente significativo, indicando que tal variável contribuiu para a elevação das concentrações de $\mathrm{SO}_{2}$ na RGV. Como mencionado por Derisio (2012), quando a temperatura aumenta na superfície pode ocorrer a chamada inversão térmica, geralmente a camada de inversão dificulta a dispersão de poluentes, ocasionando o aumento da concentração destes junto ao solo, já que nesse período de inversão os ventos horizontais geralmente têm baixa velocidade.

\section{Considerações Finais}

Este trabalho objetivou avaliar os impactos das variáveis meteorológicas temperatura, a umidade relativa, a velocidade do vento e a precipitação na probabilidade de ocorrência de picos/episódios de concentração de poluentes na RGV, Espírito Santo, Brasil, por meio do modelo Logit, no período de 01/01/2012 a 31/12/2014. Para avaliar os efeitos marginais, a qualidade do ar, no que se refere aos poluentes PTS, $\mathrm{MP}_{10}, \mathrm{SO}_{2}$, $\mathrm{CO}, \mathrm{NO}_{2}$ e $\mathrm{O}_{3}$, foi classificada como "boa" e "não boa".

A análise dos resultados mostrou que as variáveis meteorológicas apresentam relação linear com as concentrações de poluentes atmosféricos e que os índices de concentrações na RGV estão associados às mudanças dessas variáveis. Constatou-se que a ocorrência de precipitação está associada à redução da probabilidade de ocorrência de qualidade do ar "não boa" de três dos cinco poluentes avaliados. Adicionalmente notou-se que a velocidade do vento interfere significativamente na concentração de $\mathrm{PM}_{10}$, $\mathrm{SO}_{2}$ e $\mathrm{NO}_{2}$ presente na atmosfera, favorecendo ou não a poluição do ar.

Conclui-se que entre as variáveis meteorológicas avaliadas, a velocidade do vento e a precipitação foram mais significativas na redução da probabilidade de ocorrência de classificação do ar como "não boa", uma vez que apresentou os maiores efeitos marginais. Desta maneira, pode-se ainda 
inferir que maiores velocidades do vento e altos volumes de chuvas contribuíram fortemente para a qualidade do ar, na região de estudo, por intensificaram o processo de dispersão e diluição de poluentes. Desta forma, o modelo Logit mostrou ser uma ferramenta estatística satisfatória para avaliar a qualidade do ar "não boa" da RGV.

Por fim, torna-se importante destacar que os efeitos da poluição do ar na saúde são detectados em diversas cidades no mundo. Porém, segundo Arbex et al. (2012), apesar de os efeitos da poluição terem sido descritos desde a antiguidade, somente com o advento da revolução industrial a poluição passou a atingir a população em grandes proporções. Dessa forma, este estudo visa contribuir como uma ferramenta para os órgãos regulamentadores na discussão de atividades de prevenção e de controle da poluição atmosférica na RGV.

\section{Referências}

ALMEIDA, M. A. I. Modelo Aditivo Generalizado (MAG) no estudo da relação entre o número de atendimentos hospitalares por causas respiratórias e a qualidade do ar. Dissertação de Mestrado, Programa de Pós-Graduação em Engenharia Ambiental do Centro Tecnológico, Universidade Federal do Espírito Santo, Vitória, 2006.

ARBEX, M. A.; SANTOS, U. P.; MARTINS, L. C.; SALDIVA, P. H. N.; PEREIRA, L. A. A.; BRAGA, A. L. F. A poluição do ar e o sistema respiratório. Jornal Brasileiro de Pneumologia, v. 38, n. 5, p. 643-655, 2012. https://doi.org/10.1590/S1806$\underline{37132012000500015}$

BRAGA, A. L. F.; PEREIRA, L. A. A.; PROCÓPIO, M.; ANDRÉ, P. A.; SALDIVA, P. H. N. Associação entre poluição atmosférica e doenças respiratórias e cardiovasculares na cidade de Itabira, Minas Gerais, Brasil. Caderno de Saúde Pública, Rio de Janeiro, v. 23, Sup 4, p.S570-S578, 2007.

BRAGA, B.; et al. Introdução à engenharia ambiental: O desafio do desenvolvimento sustentável. 2. ed, São Paulo: Pearson Prentice Hall, 2005.

CANÇADO, J. E. D. et al. Repercussões clínicas da exposição à poluição atmosférica. J bras pneumol, v. 32, n. Supl 1, p. S5-S11, 2006.

CHIQUETTO, J. B.. Padrões atmosféricos associados a concentrações de ozônio troposférico na região metropolitana de São Paulo. Tese (Doutorado em Geografia Física) - São Paulo: Universidade de São Paulo, 2008. 
CURTIS, L.; REA, W.; SMITH-WILLIS, P.; FENYVES, E.; PAN, YAQIN. Adverse health effects of outdoor air pollutants. Environment International, v. 32, n. 6, p. 815830, 2006. https://doi.org/10.1016/i.envint.2006.03.012

DERISIO, J. C. Introdução ao controle de poluição ambiental. 4 ed. São Paulo: Oficina de Textos, 2012.

ECOSOFT CONSULTORIA E SOFTWARES AMBIENTAIS (ECOSOFT). Inventário de emissões atmosféricas da região da grande vitória. Acordo de Cooperação Técnica IEMA \& EcoSoft $\mathrm{n}^{\circ}$ 010/2009. Vitória, 2011. Disponível em: < http://www.meioambiente.es.gov.br/download/RTC10131_R1.pdf>. Acesso em: 15 de julho de 2016.

FREITAS, C. U.; LEON, A. P.; JUNGER, W.; GOUVEIA, N. Poluição do ar e impactos na saúde em Vitória, Espírito Santo. Revista de Saúde Pública, v. 50, n. 4, p. 1-9, 2016.

GEHRING, U., et al. Air pollution exposure and lung function in children: the escape project. Environmental Health Perspectives, v. 121, n. 11-12, p. 1357-1364, 2013. https://doi.org/10.1289/ehp.1306770

GOUVEIA. N.; MENDONÇA, G. A. S.; LEON, A. P.; CORREIA, J. E. M.; JUNGER, W. L.; FREITAS, C. U.; DAUMAS, R. P.; MARTINS, L. C.; GIUSSEPE, L.; CONCEIÇÃO, G. M. S.; MANERICH, A.; CUNHA-CRUZ, J.; Poluição do ar e efeitos na saúde nas populações de duas grandes metrópoles brasileiras. Epidemial Serv. Saúde, v. 12, p. 29-40, 2003.

GUJARATI, D. N.; PORTER, D. C. Basic Econometrics. 5 ed. New York: McGrawHill/Irwin, 2008.

HILL, R. C.; JUDGE, G. G.; GRIFFITHS, W. E. Econometria. 3 ed. São Paulo: Saraiva, 2010 .

HOLGATE. S. T.; SAMET. J. M.; KOREN. H. S.; MAYNARD. R. L. Air Pollution and Health. San Diego, EUA: Academic Press, 1999.

IBGE, Instituto Brasileiro de Geografia e Estatística: Censo 2010. Disponível em: $<$ http://www.ibge.gov.br/home/estatistica/populacao/censo2010/resultados_dou/ES20 10.pdf>. Acesso em: 01 agosto de 2016.

IKEFUTI, Priscilla V.; BARROZO, Ligia V.; BRAGA, Alfésio LF. Mean air temperature as a risk factor for stroke mortality in São Paulo, Brazil. International journal of biometeorology, p. 1-8, 2018.

INSTITUTO ESTADUAL DE MEIO AMBIENTE E RECURSOS HÍDRICOS DO ESTADO DO ESPÍRITO SANTO. Relatório da qualidade do ar da Região da Grande Vitória. Vitória, 2013. Disponível em: <http://www.meioambiente.es.gov.br>. Acesso em: 10 de junho de 2016.

JUNGER, W. L.; LEON, A. P. Imputation of missing data in time series for air pollutants. Atmospheric Environment, v. 102, p. 96-104, 2015. https://doi.org/10.1016/i.atmosenv.2014.11.049

KUCHENHO, H.; THAMERUS, M. Extreme value analysis of Munich air pollution data. Environmental and Ecological Statistics, v. 3, n. 2, p. 127-141, 1996. https://doi.org/10.1007/BF02427858

LEITE, R. C. M.; GUIMARÃES, E. C.; LIMA, E. A. R. L.; BARROZO, M. A. S. B.; TAVARES, M. Utilização de regressão logística simples na verificação da qualidade do ar atmosférico de Uberlândia. Engenharia Sanitária e Ambiental, v. 16, n. 1, p. 175-180, 2011. https://doi.org/10.1590/S1413-41522011000200011 
LIRA, R. S. Modelagem e previsão da qualidade do ar na cidade de Uberlândia MG. 152f. Tese (Doutorado em Engenharia Química) - Faculdade de Engenharia Química, Universidade Federal de Uberlândia, Uberlândia, 2009.

LIU, P. W. G.; JOHNSON, R. Forecasting peak daily ozone levels: part 2. A regression with time series errors model having a principal component trigger to forecast 1999 and 2002 ozone levels. Journal of the Air \& Waste Management Association, v. 53, n. 12 , p. $1472-1489,2002$. https://doi.org/10.1080/10473289.2003.10466321

LOGAN, W. P. D. et al. Mortality in the London fog incident, 1952. Lancet, p. 336-338, 1953.

LYRA, G. B.; ODA-SOUZA, M.; VIOLA, D. N. Modelos lineares aplicados à estimativa da concentração do material particulado $\left(\mathrm{PM}_{10}\right)$ na cidade do Rio de Janeiro, RJ. Revista Brasileira de Meteorologia, v. 26, n. 3, p. 392-400, 2011.

MARTINS, L. C.; LATORRE, M. R. D. O.; CARDOSO, M. R. A.; GONÇALVES, F. L. T.; SALDIVA, P. H. N.; BRAGA, A. L. F. Poluição atmosférica e atendimentos por pneumonia e gripe em São Paulo, Brasil. Revista de Saúde Pública, v. 36, n. 1, p. 88-94, 2002. https://doi.org/10.1590/S0034-89102002000100014

MENDES, C. A. B.; VEGA, F. A. C. Técnicas de regressão logística aplicada à análise ambiental. Revista Geografia (Londrina), v. 20, n. 1, p. 5-30, 2011.

MONTE, E. Z.; ALBUQUERQUE, T. T. A.; REISEN, V. A.. Impactos das Variáveis Meteorológicas na Qualidade do Ar da Região da Grande Vitória, Espírito Santo, Brasil. Rev. bras. meteorol., São Paulo, v. 31, n. 4, supl. 1, p. 546-554, dez. 2016. http://dx.doi.org/10.1590/0102-7786312314b20150100.

MOREIRA, D. M.; TIRABASSI, T.; MORAES, M. R. Meteorologia e poluição atmosférica. Ambiente e Sociedade. v. 11, n. 1, p. 1-13, 2008. https://doi.org/10.1590/S1414$\underline{753 \times 2008000100002}$

MORETTIN, P. A.; BUSSAB, W. O. Estatística básica. Saraiva, 2017.

NASCIMENTO, L. F. C.; PEREIRA, L. A. A.; BRAGA, A. L. F.; MÓDOLOA, M. C. C.; CARVALHO, J. A. C. Efeitos da poluição atmosférica na saúde infantil em São José dos Campos, SP. Revista de Saúde Pública, v. 40, p. 77-82, 2006. https://doi.org/10.1590/S0034-89102006000100013

OSTRO, B.; SANCHES, J. M.; ARANDA, C.; ESKELAND, G. S. Air pollution and mortality: results from a study of Santiago, Chile. Journal of Exposure Analysis and Environmental Epidemiology, v. 6, p. 97-114, 1996.

R CORE TEAM. R: A language and environment for statistical computing. R Foundation for Statistical Computing, Vienna, Austria, 2016. ISBN 3-900051-07-0, URL http://www.R-project.org/

SILVA, W. S.; PAIXÃO, A. N.; ARAÚJO, A. F. V.; PICANÇO, A. P. Avaliação dos benefícios da coleta de lixo em Palmas, Tocantins: uma aplicação do método de avaliação contingente. Engenharia Sanitária e Ambiental, v. 16, n. 2, p. 141-148, 2011. https://doi.org/10.1590/S1413-41522011000200007

SOUZA, J. B.; REISEN, V. A.; SANTOS, J. M.; FRANCO, G. C. Componentes principais e modelagem linear generalizada na associação entre atendimento hospitalar e poluição do ar. Revista de Saúde Pública, v. 48, n. 3, p. 451-458, 2014. https://doi.org/10.1590/S0034-8910.2014048005078

SOUZA, Juliana B. et al. Generalized additive models with principal component analysis: an application to time series of respiratory disease and air pollution data. Journal 
of the Royal Statistical Society: Series C (Applied Statistics), v. 67, n. 2, p. 453-480, 2018. https://doi.org/10.1111/rssc.12239

VARDOULAKIS, S.; KASSOMENOS, P. Sources and factors affecting $\mathrm{PM}_{10}$ levels in two European cities: implications for local air quality management. Atmospheric $\begin{array}{lllllll}\text { Environment, } & \text { v. } \quad 42, \quad \text { n. } \quad 17, \quad \text { p. } & 3949-3963,\end{array}$ https://doi.org/10.1016/j.atmosenv.2006.12.021 